\title{
Identification of a Point Mutation in the Adenosine Deaminase Gene Responsible for Immunodeficiency
}

\author{
David T. Bonthron, Alexander F. Markham, David Ginsburg, and Stuart H. Orkin \\ Divisions of Hematology-Oncology, Children's Hospital Medical Center and Brigham and Women's Hospital, Boston, Massachusetts \\ 02115; and Imperial Chemical Industries, Pharmaceuticals Division, Mereside, Alderly Park, \\ Macclesfield, Cheshire SK10 4TG, England
}

\begin{abstract}
Deficiency of adenosine deaminase (ADA) is the cause of an autosomal recessive form of immunodeficiency. We sought to define, at a molecular level, the mutations responsible for ADA deficiency in the cell line GM-1715, derived from an immunodeficient patient. Full-length complementary DNA (cDNA) for ADA was synthesized and cloned from the cell line. Sequence analysis of the clones revealed a point mutation in codon 101 (CGG to CAG) that predicts an amino acid change from arginine to glutamine. Southern blot analysis, based on silent polymorphisms in the CDNA sequence, indicated that only one of the defective alleles of the GM-1715 line had been sequenced. The mutation that was identified appears to be responsible for the loss of function in this allele, since the predicted primary structure of the enzyme is otherwise entirely normal.
\end{abstract}

\section{Introduction}

Deficiency of the enzyme adenosine deaminase (ADA) ${ }^{1}$ (EC 3.5.4.4) is responsible for approximately one-third of the cases of recessively inherited severe combined immune deficiency (SCID) (for review see reference 1). Most SCID patients manifest combined $T$ cell and $B$ cell failure, though partial ADA deficiency may allow the relative sparing of humoral immunity. Structural changes in the ADA protein, rather than regulatory mutations, may be responsible for most cases of ADA deficiency, since ADA messenger RNA (mRNA) is usually detectable in cell lines from ADA-deficient patients at levels equaling or exceeding those in normal cells (2-4). Also, structural analysis of mRNA from a number of SCID cell lines revealed abnormalities in only $\sim 10 \%$ of mRNAs examined, suggesting the presence of point mutations in the remainder (5). So far, none of the mutations causing ADA deficiency has been precisely characterized.

Address reprint requests to Dr. Orkin, Division of HematologyOncology, 320 Longwood Ave., Boston, MA 02115.

Received for publication 23 May 1985.

1. Abbreviations used in this paper: ADA, adenosine deaminase; kb, kilobase; SCID, severe combined immune deficiency.

J. Clin. Invest.

(c) The American Society for Clinical Investigation, Inc.

0021-9738/85/08/0894/04 $\$ 1.00$

Volume 76, August 1985, 894-897
We report here the sequence of ADA complementary DNA (cDNA) from a cell line (GM-1715) derived from a patient with partial ADA deficiency and impaired $T$ cell immunity. This patient's B cell function has been reported to be normal (6). A point mutation resulting in a nonconservative amino acid change appears to be the cause of a loss of function in one ADA allele in this patient.

\section{Methods}

The lymphoid cell line GM-1715 (Human Genetic Mutant Cell Repository, Camden, NJ) was maintained in RPMI 1640 medium supplemented with $10 \%$ fetal calf serum.

Total RNA was prepared from cells by lysis in buffer containing 6 $M$ guanidine hydrochloride. Poly $\mathrm{A}^{+}$RNA was isolated by oligo-(dT) cellulose chromatography (7). cDNA was synthesized by the method of Okayama and Berg (8), as modified by Gubler and Hoffman (9), blunt-ended with T4 polymerase, and ligated to synthetic EcoRI linkers after methylation of internal EcoRI sites. After it was digested with EcoRI, cDNA was purified from free linkers by Sepharose CL4-B chromatography and ligated into EcoRI-digested alkaline phosphatasetreated lambda gt11 DNA (10). Benton-Davis screening of the library of 375,000 phage clones was performed by standard methods (7), using as a probe a previously characterized 1.3-kilobase (kb) ADA cDNA insert (from ADA clone $1 \mathrm{~b}$ in reference 19).

For further analysis, the inserts of positive gt 11 clones were subcloned into the plasmid pBR 322 and then into M13 mpl1 (11). A series of oligonucleotides complementary to the coding strand of the previously published human ADA cDNA sequence $(2,12)$ was synthesized by the modified phosphotriester method (13), either manually or on a DNA synthesizer (model SAM1; Biosearch, San Rafael, CA). Their sequences are given in Table I and their positions along the mRNA are shown in Fig. 1. These oligonucleotides were used for internal priming of sequencing reactions $(14,15)$ on the $M 13$ subclones of ADA cDNAs from the GM-1715 cell line. An M13 universal primer (New England Biolabs, Beverly, MA) was used to complete the $3^{\prime}$ ends of the sequences.

For Southern blot analysis (16), total genomic DNA was prepared from cultured cells or from normal human blood by the method of Bell et al. (17), and after digestion and electrophoresis, transferred to GeneScreen Plus membrane (New England Nuclear, Boston, MA). DNA fragments used as probes were cut from low-melt agarose gels and labeled with large fragments of DNA polymerase I by random oligonucleotide priming (18).

\section{Results}

For rapid sequencing of the ADA cDNAs, we adopted a strategy that is generally applicable both for the characterization of further ADA cDNAs and for the sequencing of any mutant cDNA for which the normal sequence is known. By priming 
Table I. Sequences of the Oligonucleotide Primers Used for Dideoxy Sequencing *

\begin{tabular}{lcl}
\hline No. & Priming site & Sequence \\
\hline 1 & 95 & 5'-GCGATCCCTCTCCTC-3' \\
2 & 246 & 5'-TCTACAAACTCATAGGC-3' \\
3 & 375 & 5'-GCCACCACCTCGTCTGG-3' \\
4 & 541 & 5'-CATCTCCAGCCAGGT-3' \\
5 & 697 & 5'-GTGTCCCAGCCGCTCTG-3' \\
6 & 843 & 5'-TGGTCATTTTTGAGCCG-3' \\
7 & 984 & 5'-AAACTAGATTTGGCCGC-3' \\
8 & 1,266 & 5'-ACATAATCAGAGAAGTG-3'
\end{tabular}

* Each is complimentary to the coding strand of ADA cDNA at the positions shown in Fig. 1.

the dideoxy sequencing reactions from internal sites along the cDNA sequence, the need for fragmentation of the clone and further subcloning of the fragments was obviated. Since this subcloning is frequently the most time-consuming step in M13 sequencing, the sequencing of a number of mutant cDNAs of the same gene can be considerably speeded up by this method.

Four independently isolated full-length cDNAs from the GM-1715 cell line were analyzed, along with a normal ADA cDNA (clone la from reference 19). One clone, designated 1715-N, was sequenced in its entirety. It shows single base changes from the published ADA cDNA sequence (2) at three positions. Two of these are silent polymorphisms (see Table II), while the remaining one, a $\mathrm{G}$ to A change at position 302 , causes the nonconservative amino acid change arginine to glutamine (see Fig. 1). As no other amino acid substitutions were predicted by the cDNA sequence, we inferred that this arginine to glutamine change is responsible for the functional deficiency of this allele.

Sequencing of further cDNA clones (1715-D, 1715-H, and 1715-L) again revealed the same three alterations in all three clones. Approximately $75 \%$ of each of these clones was sequenced. No differences were found among the four cDNA clones from GM-1715. Sequencing of the normal (and demonstrably functional) ADA cDNA (19) incidentally revealed one further silent polymorphism, a $\mathrm{C}$ to $\mathrm{T}$ change at position 273 . This sequence otherwise conformed to that in reference 2 with none of the alterations seen in the 1715 clones.

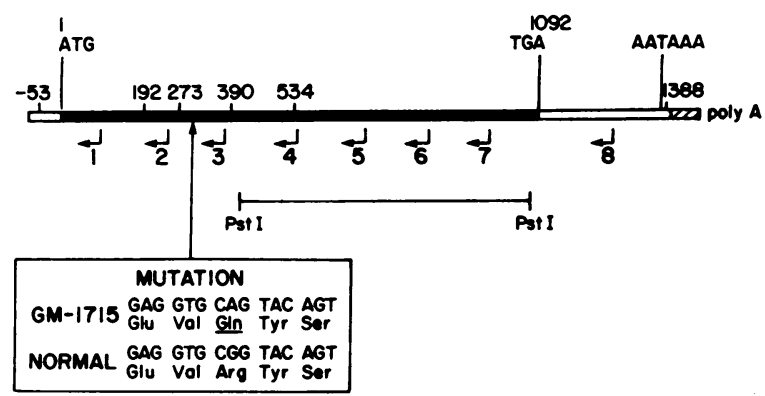

Figure 1. Linear representation of ADA cDNA with positions of mutation, polymorphisms (above the line), and oligonucleotide sequencing primers (numbered 1-8). The coding region is shown in black. The numbering of the nucleotide sequence is as in reference 5 . The 0.65-kb Pst I cDNA fragment used for Southern blot analysis of the Bal I polymorphism at position 534 is shown by the bar.
Table II. Summary of Sequence Polymorphisms Identified in the ADA $C D N A$, with Sources*

\begin{tabular}{lllllll}
\hline Position & & & & & & \\
\hline-53 & C & A & A & A & A & A \\
192 & G & G & A & A & G & G \\
273 & C & C & C & T & C & C \\
390 & G & A & G & G & G & G \\
534 & G & G & A & A & G & G \\
1,388 & G & A & A & A & A & A \\
Clone & ADA33 & ADA211 & & ADA1a & 1715 & \\
Reference & $(5)$ & $(5)$ & (2) & (this work) & (20)
\end{tabular}

* Numbering is as in reference 5 .

Comparison of the present sequences with the published sequence from our laboratory (2) revealed one error in the $3^{\prime}$ untranslated region. Rereading of the original autoradiographs confirmed the true sequence to be 1284-CCCTGGCCAGG1295, in agreement with that of references 5 and 20. Further differences between the published sequences occurred in the 5 untranslated region. All of our clones, including those from GM-1715, had an extra $C$ between positions -12 and -11 of the sequence of Adrian et al. (GGGGCGCACGAG). This is in agreement with the sequence of Valerio et al. (20). However, the latter sequence, when compared with all others, had an inversion of two bases at positions -23 and -22 (Valerio et al., GGGAGCGACGCCGGGG; all other sequences, GGGAGCAGCGCCGGGG).

The silent $A$ to $G$ change at position 534 in our 1715 ADA cDNA clones creates a new cleavage site for the restriction enzyme Bal I. To address the question of whether GM-1715 is homozygous for the allele whose cDNA we sequenced, Southern blot analysis of Bal I-digested genomic DNAs was performed, using as probe an internal Pst I fragment of cDNA spanning this site (see Fig. 1). This showed GM-1715, as well as the commonly used T cell line MOLT 4, to be heterozygous for this polymorphism (Fig. 2).

\section{Discussion}

The mutations causing ADA deficiency are known to be heterogeneous $(4,5,21)$, but to date none has been defined at the sequence level. In most ADA-deficient cell lines, ADA

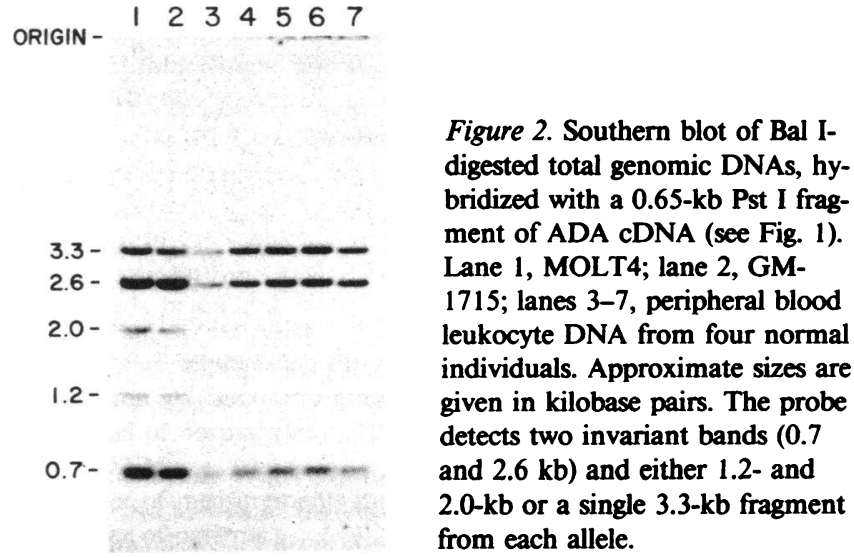


mRNA is present in normal or supranormal amounts (2-4), but enzyme activity and immunologically detectable protein levels are greatly diminished $(22,23)$. The majority of the mutations are therefore thought to lie within the protein coding sequence. Since the ADA mRNA in ADA-deficient cell lines can often be translated in vitro into normal-sized ADA protein $(3,24)$, it can be inferred that the mutant ADA proteins are sometimes unstable in vivo. Recently, Adrian et al. (5) performed $\mathrm{S} 1$ nuclease analysis on mRNA from seven ADAdeficient cell lines. Four of these showed patterns indistinguishable from those of normal cells. In the remaining three, structural aberrations in the mRNA were detected. It was concluded that point mutations are probably responsible for most, though not all, cases of ADA deficiency. GM-1715 was not examined.

We report here the cloning and sequence analysis of fulllength cDNAs from an ADA-deficient cell line. Four apparently identical, though independently isolated, clones have been sequenced. In each, a $\mathrm{G}$ to $\mathrm{A}$ mutation at base number 302 of the coding sequence was found, which predicts a glutamine residue to be substituted for arginine in the ADA protein at codon 101. Since no other abnormalities were found in the sequence, we conclude that this mutation is the one responsible for loss of function of this allele. Because all four of the clones we have sequenced appeared identical, we considered that GM-1715 might be homozygous for a single mutant ADA allele. However, the Southern blot analysis indicated that this line is heterozygous for a Bal I restriction fragment length polymorphism at the site of residue 534. Thus, only one of the mutant ADA alleles from GM-1715 has been analyzed.

The lymphoid cell line GM-1715 and the fibroblast line GM-2445 were derived from the same patient with ADA deficiency (6). It has been suggested previously that this patient's phenotype may have resulted from underproduction of a normal enzyme rather than from any abnormality of protein structure, since a spontaneous reversion to normal enzyme levels apparently occurred on prolonged culture of cells from the patient (6). Furthermore, ADA from the original fibroblast line GM-2445, while present at $<3 \%$ of normal levels, has approximately normal calculated specific activity, in contrast to the reduced specific activity of ADA from most other ADA-deficient cell lines (22). However, Northern blotting has previously shown that GM-1715 contains normal to above normal levels of ADA mRNA $(2,3)$. Down-regulation of genes coding for structurally normal proteins is therefore unlikely to account entirely for this patient's ADA deficiency. Although there may be other explanations for this apparent paradox, the following model may be considered. The normal level of ADA mRNA in GM-1715 is derived mostly from the allele whose cDNA we have sequenced and directs the synthesis of an extremely unstable (or non-cross-reactive) protein, which is not detected enzymatically or immunologically in GM2445. The other allele is down-regulated by a cis-acting mutation, which may explain our failure to clone cDNA corresponding to this allele from GM-1715. The low level of ADA produced from this allele would be of normal specific activity, as observed in GM-2445. Phenotypic reversion to normal enzyme levels would presumably have occurred by activation of this second allele.

A number of polymorphic sites in the ADA cDNA sequence have been noted in the present study. All but one of these can be recognized by comparison of the previously published sequences $(5,12,20)$, which differ at these positions. Polymorphisms such as that giving rise to the new Bal I restriction site in GM-1715 will distinguish between alleles and facilitate identification of cDNAs that probably differ with respect to their mutations. This work demonstrates that a structural change in the ADA sequence may cause ADA deficiency. Further sequence analysis of other mutant alleles will permit detailed mapping of sites in the ADA protein that are critical to enzyme function and stability in vivo.

\section{Acknowledgments}

We thank R. Neve for assistance with cDNA cloning.

This work was supported by grants from the National Institutes of Health and the March of Dimes Foundation.

\section{References}

1. Martin, D. W., and E. W. Gelfand. 1981. Biochemistry of diseases of immunodevelopment. Annu. Rev. Biochem. 50:845-877.

2. Daddona, P. E., D. S. Shewach, W. N. Kelley, P. Argos, A. F. Markham, and S. H. Orkin. 1984. Human adenosine deaminase. cDNA and complete primary amino acid sequence. J. Biol. Chem. 259:12101-12106.

3. Valerio, D., M. G. C. Duyvesteyn, H. van Ormondt, P. Meera Khan, and A. J. van der Eb. 1984. Adenosine deaminase deficiency in cells derived from humans with severe combined immunodeficiency is due to an aberration of the ADA protein. Nucleic Acids Res. 12:10151024.

4. Daddona, P. E., B. L. Davidson, J-L. Perignon, and W. N. Kelley. 1985. Genetic expression in partial adenosine deaminase deficiency. J. Biol. Chem. 260:3875-3880.

5. Adrian, G. S., D. A. Wiginton, and J. J. Hutton. 1984. Structure of adenosine deaminase mRNAs from normal and adenosine deaminase deficient human cell lines. Mol. Cell. Biol. 4:1712-1717.

6. Uberti, J., W. D. Peterson, J. J. Lightbody, and R. M. Johnson. 1983. A phenotypically normal revertant of an adenosine deaminase deficient lymphoblast cell line. J. Immunol. 130:2866-2870.

7. Maniatis, T., E. F. Fritsch, and J. Sambrook. 1982. Molecular Cloning: A Laboratory Manual. Cold Spring Harbor Laboratory, Cold Spring Harbor, NY.

8. Okayama, H., and P. Berg. 1982. High-efficiency cloning of fulllength cDNA. Mol. Cell. Biol. 2:161-170.

9. Gubler, U., and B. J. Hoffman. 1983. A simple and very efficient method for generating cDNA libraries. Gene (Amst.). 25:263-269.

10. Young, R. A., and R. W. Davis. 1983. Efficient isolation of genes by using antibody probes. Proc. Natl. Acad. Sci. USA. 80:11941198.

11. Messing, J. 1983. New M13 vectors for cloning. Methods Enzymol. 101:20-78.

12. Orkin, S. H., P. E. Daddona, D. S. Shewach, A. F. Markham, G. A. Bruns, S. C. Goff, and W. N. Kelley. 1983. Molecular cloning of human adenosine deaminase gene sequences. J. Biol. Chem. 258: 12753-12756.

13. Markham, A. F., M. D. Edge, T. C. Atkinson, A. R. Greene, G. R. Heathcliffe, C. R. Newton, and D. Scanlon. 1980. Solid phase phosphotriester synthesis of large oligodeoxyribonucleotides on a polyamide support. Nucleic Acids Res. 8:5193-5205.

14. Sanger, F., S. Nicklen, and A. R. Coulson. 1977. DNA sequencing with chain-terminating inhibitors. Proc. Natl. Acad. Sci. USA. 74:5463-5467.

15. Biggin, M. D., T. J. Gibson, and G. F. Hong. 1983. Buffer gradient gels and ${ }^{35} \mathrm{~S}$ label as an aid to rapid DNA sequence determinations. Proc. Natl. Acad. Sci. USA. 80:3963-3965.

16. Southern, E. M. 1975. Detection of specific sequences among DNA fragments separated by electrophoresis. J. Mol. Biol. 98:503517. 
17. Bell, G. I., J. H. Karam, and W. J. Rutter. 1981. Polymorphic DNA regions adjacent to the 5 ' end of the human insulin gene. Proc. Natl. Acad. Sci. USA. 78:5759-5763.

18. Feinberg, A. P., and B. J. Hoffman. 1983. A technique for radiolabelling DNA restriction endonuclease fragments to high specific activity. Anal. Biochem. 132:6-13.

19. Orkin, S. H., S. C. Goff, W. N. Kelley, and P. E. Daddona. 1985. Transient expression of human adenosine deaminase cDNAs: identification of a nonfunctional clone resulting from a single aminoácid substitution. Mol. Cell. Biol. 5:762-767.

20. Valerio, D., M. G. C. Duyvesteyn, B. M. M. Dekker, G. Weeda, Th. M. Berkvens, L. van der Voorn, H. van Ormondt, and A. J. van der Eb. 1985. Adenosine deaminase: characterization and expression of a gene with a remarkable promoter. EMBO (Eur. Mol. Biol. Organ.) J. 4:437-443.
21. Hirschhorn, R., F. Martiniuk, V. Roegner-Maniscalco, J.-L Perignon, and T. Jenkins. 1983. Genetic heterogeneity in partial adenosine deaminase deficiency. J. Clin. Invest. 71:1887-1892.

22. Daddona, P. E., M. A. Frohman, and W. N. Kelley. 1980. Human adenosine deaminase and its binding protein in normal and adenosine deaminase deficient fibroblast cell strains. J. Biol. Chem. 255:5681-5687.

23. Wiginton, D. A., and J. J. Hutton. 1982. Immunoreactive protein in adenosine deaminase deficient human lymphoblast cell lines. J. Biol. Chem. 257:3211-3217.

24. Adrian, G. S., arid J. J. Hutton. 1983. Adenosine deaminase messenger RNAs in lymphoblast cell lines derived from leukemic patients and patients with hereditary adenosine deaminase deficiency. J. Clin. Invest. 71:1649-1660. 